\title{
Availability of CD10 Immunohistochemistry as a Marker of Breast Myoepithelial Cells on Paraffin Sections
}

\author{
Suzuko Moritani, M.D., Ph.D., Ryoji Kushima, M.D., Ph.D., Hiroyuki Sugihara, M.D., Ph.D., \\ Masamichi Bamba, M.D., Ph.D., Tadao K. Kobayashi, Ph.D., C.F.I.A.C., Takanori Hattori, M.D., Ph.D. \\ Department of Pathology, Shiga University of Medical Science (SM, RK, HS, MB, TH), Ohtsu and \\ Department of Pathology, Saiseikai Shiga Hospital (SM, TKK), Imperial Gift Foundation, Ritto, Japan
}

CD10, also called common acute lymphoblastic leukemia antigen (CALLA), was recently found to be expressed in nonhematopoietic tissues. Although CD10 was also identified in human breast myoepithelial cells, its availability of immunohistochemistry on paraffin sections has not been examined so far. In the present study, we demonstrated CD10 immunohistochemically on paraffin sections of both normal and pathological breast tissues, comparing its staining patterns to those of smooth muscle actin (SMA), which is now commonly used to highlight myoepithelium. CD10 was consistently positive in normal breast myoepithelial cells. CD10 also clearly highlighted myoepithelial cells in intraductal papilloma, adenosis, ductal hyperplasia, fibroadenoma, and phyllodes tumor as well as SMA did. In atypical ductal hyperplasia and ductal carcinoma in situ, continuous, discontinuous, and totally negative stainings of both CD10 and SMA were noted, depending on foci of neoplastic cell nests. However, both stainings clearly demonstrated myoepithelial cells of cancerized acini, being useful in differentiating lobular cancerization from microinvasion. Because SMA was also positive in normal vessels and spindle-shaped stromal cells, CD10, which was negative in vessels, was useful in differentiating myoepithelial cells from thin vascular wall in intracystic lesions with delicate papillae. Although background staining of spindle-shaped stromal cells was also noted in $\mathrm{CD10}$, the positive cell number was less, and the signal was weaker than that of SMA. The absence of myoepithelial cells in invasive ductal carcinomas was more clearly highlighted by CD10 than SMA. We concluded that CD10 could be another useful marker of breast myoepithelial cells on paraffin sections. Combination of

Copyright (C) 2002 by The United States and Canadian Academy of Pathology, Inc

VOL. 15, NO. 4, P. 397, 2002 Printed in the U.S.A.

Date of acceptance: October 29, 2001.

Address reprint requests to: Suzuko Moritani, Department of Pathology,

Shiga University of Medical Science, Seta-tsukinowa-cho, Ohtsu, Shiga, 520-2192, Japan; fax: +81-77-543-9880.
CD10 and SMA will provide more sophisticated information about presence or absence of myoepithelial cells in confusing breast lesions.

KEY WORDS: Breast, CD10, Immunohistochemistry, Myoepithelial cell, Paraffin section, Smooth muscle actin.

Mod Pathol 2002;15(4):397-405

Ducts and lobuloalveolar structures of the normal breast consist of two distinct cell types. One is the inner layer of epithelial cells lining the lumen, and the other is the outer layer of myoepithelial cells, showing the characteristic two-cell pattern (1). As the latter cells are rich in myofibrils in their cytoplasm, they are contractile and sensitive to oxytocin. The appearance of myoepithelial cells on hematoxylin and eosin sections varies considerably depending on both physiological and pathological status (1).

Presence of this two-cell pattern in the glandular structures in some pathological situations is generally an important histological hallmark of benignity. There are many benign lesions that show prominent ductal proliferation, causing misinterpretations of such lesions as malignancy. Of these, adenosis and intraductal papilloma with sclerosis are sometimes difficult to differentiate from invasive ductal carcinoma (2). Distinction between some intracystic papillary carcinomas $(3,4)$ with low nuclear grade and intraductal papilloma are challenging. A two-cell pattern of epithelial and myoepithelial cells is maintained even in such problematic lesions, except in one special type of adenosis called "microglandular adenosis" $(5,6)$. Because it is not always easy to identify myoepithelial cells on routine hematoxylin and eosin sections, immunohistochemical demonstration of myoepithelial cells is helpful to make exact diagnoses of these lesions. Smooth muscle actin (SMA) is commonly used to highlight myoepithelial cells (7).

CD10, also called common acute lymphoblastic leukemia antigen (CALLA), has mainly been applied 
to the diagnosis of hematopoietic malignancies. CD10 was recently reported also to be expressed in various nonhematopoietic cells (8-10), and immunohistochemistry in paraffin sections became useful in differential diagnosis in some neoplasms (1114). CD10 was also identified as the same molecule as neutral endopeptidase, which can inactivate oxytocin and some other peptide hormones (15).

CD10 was also identified in myoepithelial cells of the human breast (16). Ultrastructural studies confirmed the presence of this antigen on the lateral membrane of the myoepithelial cells (16). Clarke et al. (17) successfully separated luminal cells from myoepithelial cells by immunomagnetic techniques, using immunohistochemical expression of epithelial membrane antigen in luminal cells and of CD10 in myoepithelial cells, respectively. In the area of diagnostic pathology of the breast, CD10 expression in myoepithelial cells was evaluated in fibroadenoma and phyllodes tumor $(9,18)$. However, these studies were performed on frozen sections. CD10 expression in myoepithelial cells has not been examined in a wide variety of breast lesions in formalin-fixed paraffin-embedded tissue sections so far.

In this study, we demonstrated CD10 expression in various breast lesions on paraffin sections, comparing its staining pattern with those of $\alpha$-smooth muscle actin (SMA) and S-100 protein, which is also a marker of myoepithelial differentiation (7), and evaluated availability of CD10 immunohistochemistry on differential diagnosis of breast lesions.

\section{MATERIALS AND METHODS}

\section{Study Cases}

Representative paraffin sections of 70 distinct lesions from 46 cases of breast examined in the Department of Pathology, Shiga University of Medical Science, Ohtsu, Japan between 1996 and 2001 were selected for the study. All the specimens were of either mastectomy or excisional biopsy, and cases of needle biopsy were excluded from the study. Specimens included were 2 ductal adenomas, 7 intraductal papillomas, 5 lesions that contained foci of adenosis, 4 lesions that contained multiple cystic glands, 6 lesions that contained foci of ductal hyperplasia, 2 lesions that contained foci of atypical ductal hyperplasia, 20 ductal carcinomas in situ (DCIS), 10 usual invasive ductal carcinomas, 2 fibroadenomas, 2 phyllodes tumors (low grade), 2 matrix-producing carcinomas, 1 adenoid cystic carcinoma, 2 mucinous carcinomas, and 1 medullary carcinoma. The patients' ages ranged from 14 to 96 years old (average, 52.3 y). Duration of formalin fixation was $<2$ days in all the cases examined.
Immunohistochemistry

We made $4-\mu \mathrm{m}$-thick serial sections from each representative paraffin block, and immunohistochemical stainings for $\alpha$-SMA, CD10, and S-100 protein were performed in this order. The primary antibodies used are listed in Table 1. For CD10 staining, the sections were immersed in distilled water and pretreated in an autoclave at $121^{\circ} \mathrm{C}$ for 25 minutes for the purpose of antigen retrieval. No pretreatment was performed for SMA and S-100. After 20 minutes' incubation with $0.3 \%$ hydrogen peroxidase, all the sections were incubated with the primary antibodies for 2 hours at room temperature, followed by signal detection using the streptavidin-biotin-peroxidase method (LSAB2 Kit/ HRP; DAKO, Kyoto, Japan).

\section{RESULTS}

\section{Normal Ducts and Lobules}

Various proportions of normal ducts and lobules were present in the same sections in 42 of 43 cases (Fig. 1A and E). In all these sections, CD10 was strongly and consistently positive in myoepithelial cells of both ducts (Fig. 1B) and lobular acini (Fig. $1 F)$. It was exclusively negative in luminal epithelial cells. The staining pattern of CD10 in myoepithelial cells was identical to that of SMA (Fig. 1C and G). Although SMA was also positive in the wall of vessels, CD10 was completely negative. S-100 protein was positive mainly in acinar myoepithelial cells (Fig. 1H) but was also positive in ductal myoepithelial cells in various proportions. It was also randomly positive in luminal epithelial cells of ducts (Fig. 1D).

\section{Cystic Glands}

Cystic glands were present in 7 cases. Large cystic glands whose lining epithelium was markedly distended showed negative or decreased staining density for both CD10 and SMA. Small to mediumsized cystic glands whose cytoplasm of the lining epithelium was still identifiable were positive for CD10 and SMA corresponding to the outer layer of these glands. S-100-positive cells were randomly scattered in luminal or myoepithelial cells whose cytoplasm was identifiable but were completely negative in the large cystic glands whose cytoplasm of lining epithelium was distended.

TABLE 1

\begin{tabular}{lcl}
\hline \multicolumn{1}{c}{ Antibody } & Source Company & \multicolumn{1}{c}{ Dilution } \\
\hline CD10 (anti CALLA, NU-N1) & Japanese Nichirei (Tokyo) & $1: 50$ \\
$\alpha$-Smooth muscle actin (1A4) & DAKO (Kyoto) & $1: 100$ \\
S-100 & DAKO (Kyoto) & $1: 100$ \\
\hline
\end{tabular}



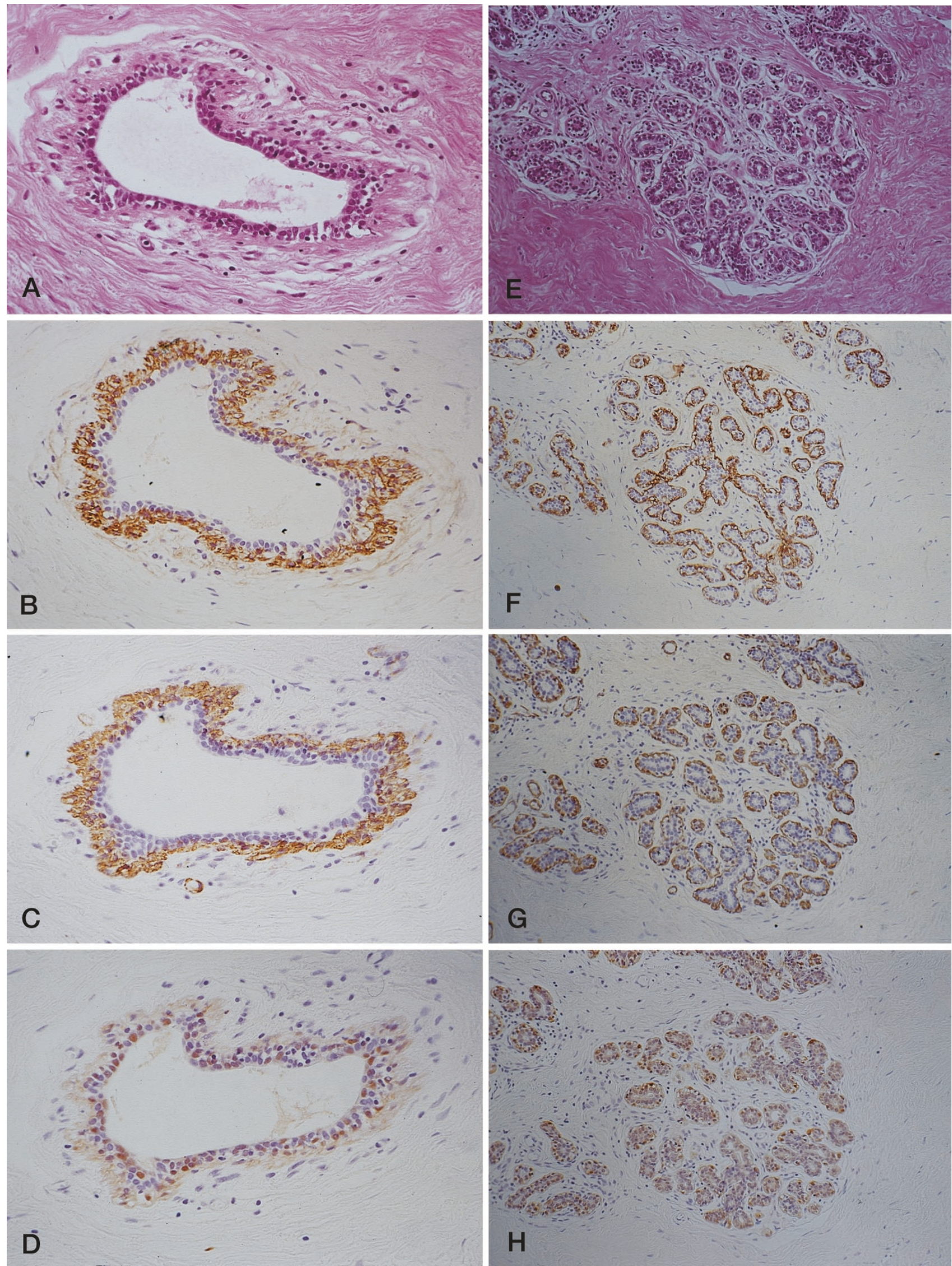

FIGURE 1. Serial sections of normal duct (A-D) and acini (E-H). Both CD10 (B, F) and SMA (C, G) were strongly and consistently positive in the myoepithelial cells. S-100 was positive in the myoepithelium of acini $(\mathbf{H})$, but it was also randomly positive in the luminal epithelium and myoepithelium of the duct. 


\section{Ductal Adenoma}

Two ductal adenomas showed heterogenous staining patterns. In one case, both CD10 and SMA were positive in $<50 \%$ of the tubular outer layer; however, S-100 was positive in most of the cells in the outer layer of proliferated tubules and also in some cells growing in solid nests. The other one case showed positive only for CD10 in normal glands; adenoma lesion, CD10, SMA, and S-100 were all negative.

\section{Adenosis}

We had five cases that contained foci of adenosis (two florid duct adenosis, three sclerosing adenosis). In four cases (one florid duct adenosis, three sclerosing adenosis), both CD10 and SMA were strongly and consistently positive in the outer layer of proliferated tubules (Fig. 2A-C). In the other one case of florid duct adenosis, the staining of SMA was weaker than that of CD10, although both were positive in the outer layer of proliferated tubules. In two cases (1 florid duct adenosis, 1 sclerosing adenosis), $80 \%$ of the outer layer of proliferated tubules was positive for S-100, but in the other three, S-100 was randomly positive in the cells of inner and outer layers.

\section{Intraductal Papilloma}

In all seven cases of intraductal papilloma, CD10 (Fig. 3B) and SMA (Fig. 3C) were positive in the cells beneath the epithelial cells that line the papillary architectures and in the outer layer of the tubules proliferating in the fibrovascular stroma. SMA was also positive in the vascular components of the stroma. One lesion contained infarcted and sclerotic areas (Fig. 3D). In the infarcted area, CD10 was positive, corresponding to the outer layer of the silhouette of necrotic tubules (Fig. 3E), indicating that the infarcted lesion had been benign when it was viable. However, the SMA was only vaguely stained in such parts (Fig. 3F). In the sclerotic area, SMA was positive in the outer layer of the atrophic tubules and vessel wall; CD10 was almost negative in these parts. One more lesion contained the foci of sclerosis, where some atrophic tubules were noted. SMA was positive in these tubules, but CD10 was negative. S-100-positive cells were generally small in number and were scattered in the outer layer or basal side of the lining epithelium and also in the stroma in individual patterns.

\section{Ductal Hyperplasia}

In all six of the lesions of ductal hyperplasia, the entire circumference of the ducts, corresponding to myoepithelial cells, was positive for CD10 and SMA.

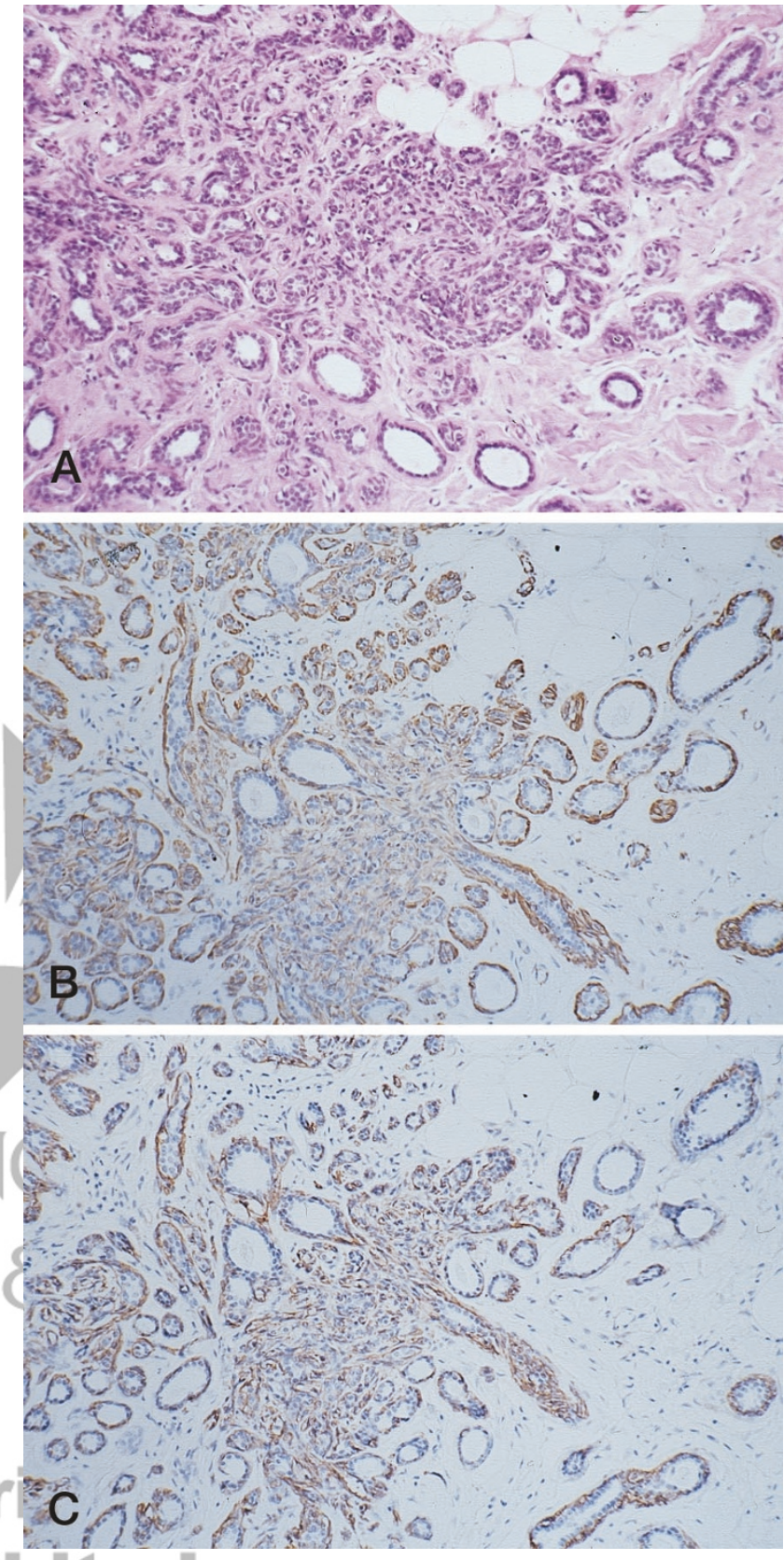

FIGURE 2. Adenosis (A). Both CD10 (B) and SMA (C) were strongly and consistently positive in the myoepithelial cells of proliferated tubules.

S-100 was randomly positive in myoepithelial cells, but most of the lesion was negative.

\section{Atypical Ductal Hyperplasia and Ductal Carcinoma In Situ}

Two cases contained foci of atypical ductal hyperplasia, which showed atypical ductal epithelial proliferation, but not sufficient to be labeled DCIS. Both lesions were associated with DCIS. In one case, the entire circumference of all the atypical ducts, corresponding to myoepithelial cells, was 

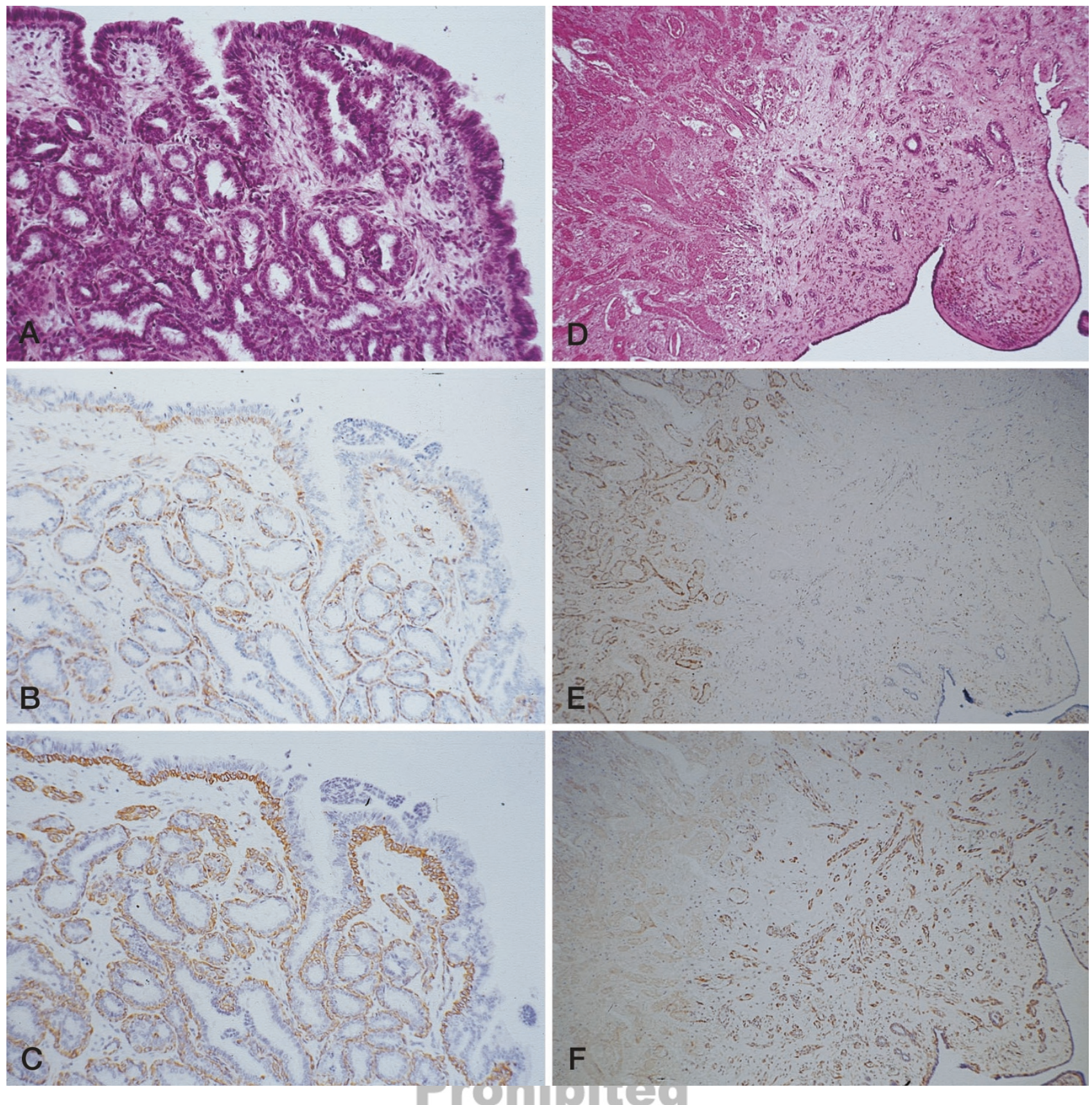

FIGURE 3. Intraductal papilloma (A, D). Both CD10 (B) and SMA (C) were strongly positive in the myoepithelial cells. CD10 also highlighted the silhouette of myoepithelium in the infarcted area (E), where SMA was negative (F). Meanwhile, SMA was positive in the vessels and myoepithelium of atrophic tubules in the sclerotic area (F), where CD10 was negative (E).

positive for CD10 and SMA. In this case, S-100 was totally negative. In the other case, 80 to $90 \%$ of the atypical ducts were CD10 and SMA positive in the entire circumference; however, the remaining ducts showed focally negative. In this case, the entire circumference of $60 \%$ of the atypical ducts was positive for S-100.

We had 20 cases of DCIS. The outer layer of neoplastic ducts, solid nests, cribriform structures, and cystically dilated ducts with papillary projection showed various staining patterns. Some lesions showed positive for both CD10 and SMA in their entire circumference; others showed discontinuously positive or entirely negative. Generally, smaller foci showed encircled pattern of positivity for CD10 and SMA. In 6 of the 20 DCIS, SMApositive parts predominated over CD10-positive parts. In foci of intralobular extension of the neoplasm (Fig. 4A), most of the acinar myoepithelial cells were clearly demonstrated by both CD10 and SMA (Fig. 4B and C). S-100 was almost negative, except for a few foci of intralobular extension of the neoplasm, where acinar myoepithelial cells were positive. 
Four of 20 DCISs were so-called intracystic papillary carcinoma, which architecturally resembled an intraductal papilloma; that is, had intracystic papillary growth with fibrovascular core (Fig. 4D). Unlike the case of intraductal papilloma, CD10positive myoepithelial cells were absent in the areas of papillary projection (Fig. 4E). SMA was positive in vessels and in some spindle-shaped stromal cells in the fibrovascular core (Fig. 4F); however, the characteristic positive pattern of myoepithelial cells seen in intraductal papillomas was not present. Some of the vascular stainings of SMA just beneath the lining of the neoplastic epithelium, especially where fibrovascular stromal cores were narrow and delicate, were confused with those of myoepithelial cells. A few S-100-positive stromal cells were scattered in the fibrovascular core.

\section{Invasive Carcinoma}

We had 17 cases of invasive carcinomas (Fig. 5A), including two matrix-producing carcinomas, 1 adenoid cystic carcinoma, one invasive micropapillary carcinoma, two mucinous carcinomas, and one
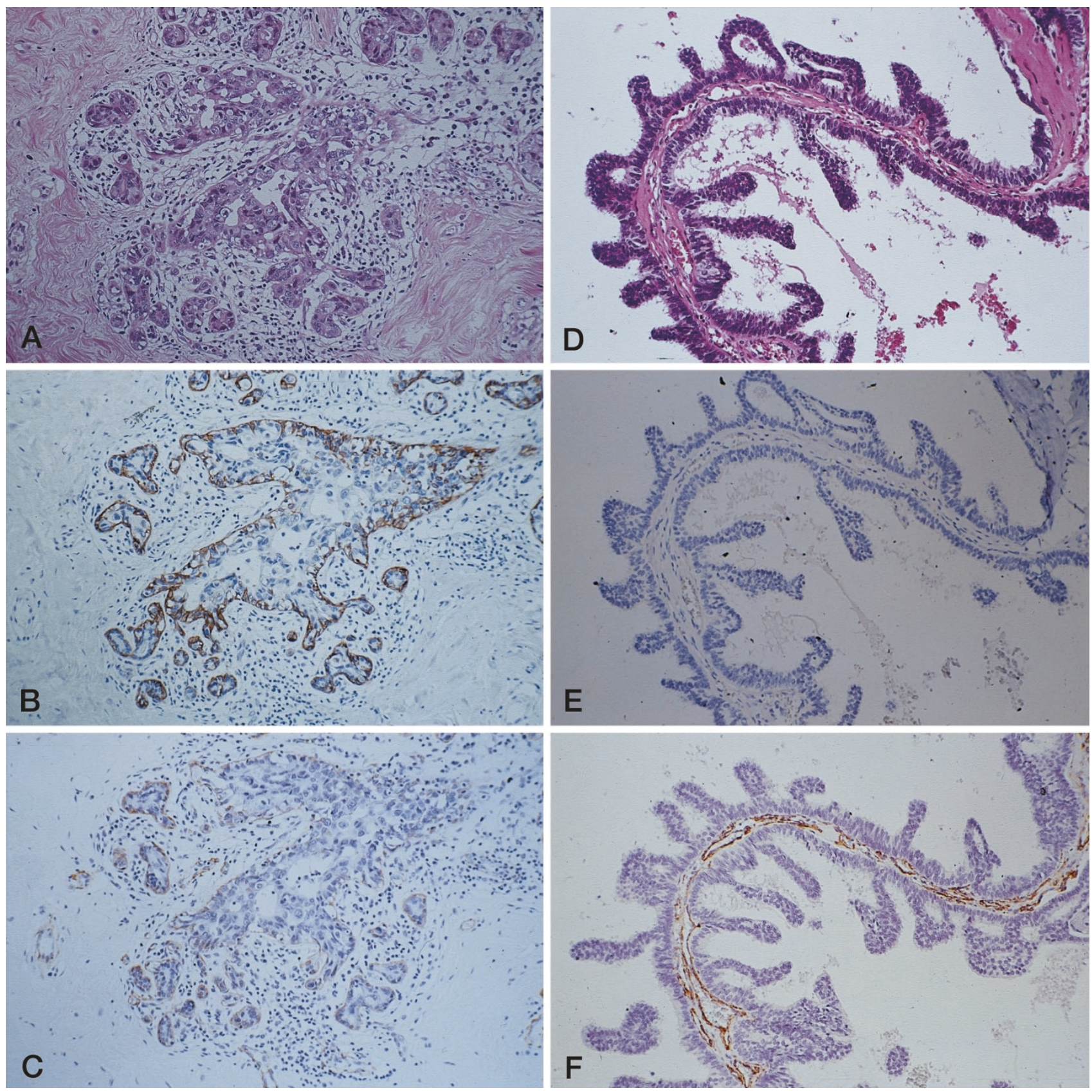

FIGURE 4. Ductal carcinoma in situ (A). Both CD10 (B) and SMA (C) highlighted myoepithelium of cancerized lobules. Intracystic papillary carcinoma (D). Fibrovascular core was negative for CD10 (E), whereas SMA was positive in the delicate vascular network (F). Vascular staining beneath the neoplastic epithelium could be confused with myoepithelium (F). 
medullary carcinoma. In all these lesions, there was no myoepithelial component that was positive for either SMA or CD10 (Fig. 5B-C); however, SMA was positive in many of the spindle cells of the desmoplastic stromal component. This background staining of stromal cells occasionally encircled the small neoplastic cell nests and tubules (Fig. 5C). CD10 was also positive in a small number of these stromal cells, but the staining was generally weak (Fig. 5B). S-100 was entirely negative in neoplastic areas.
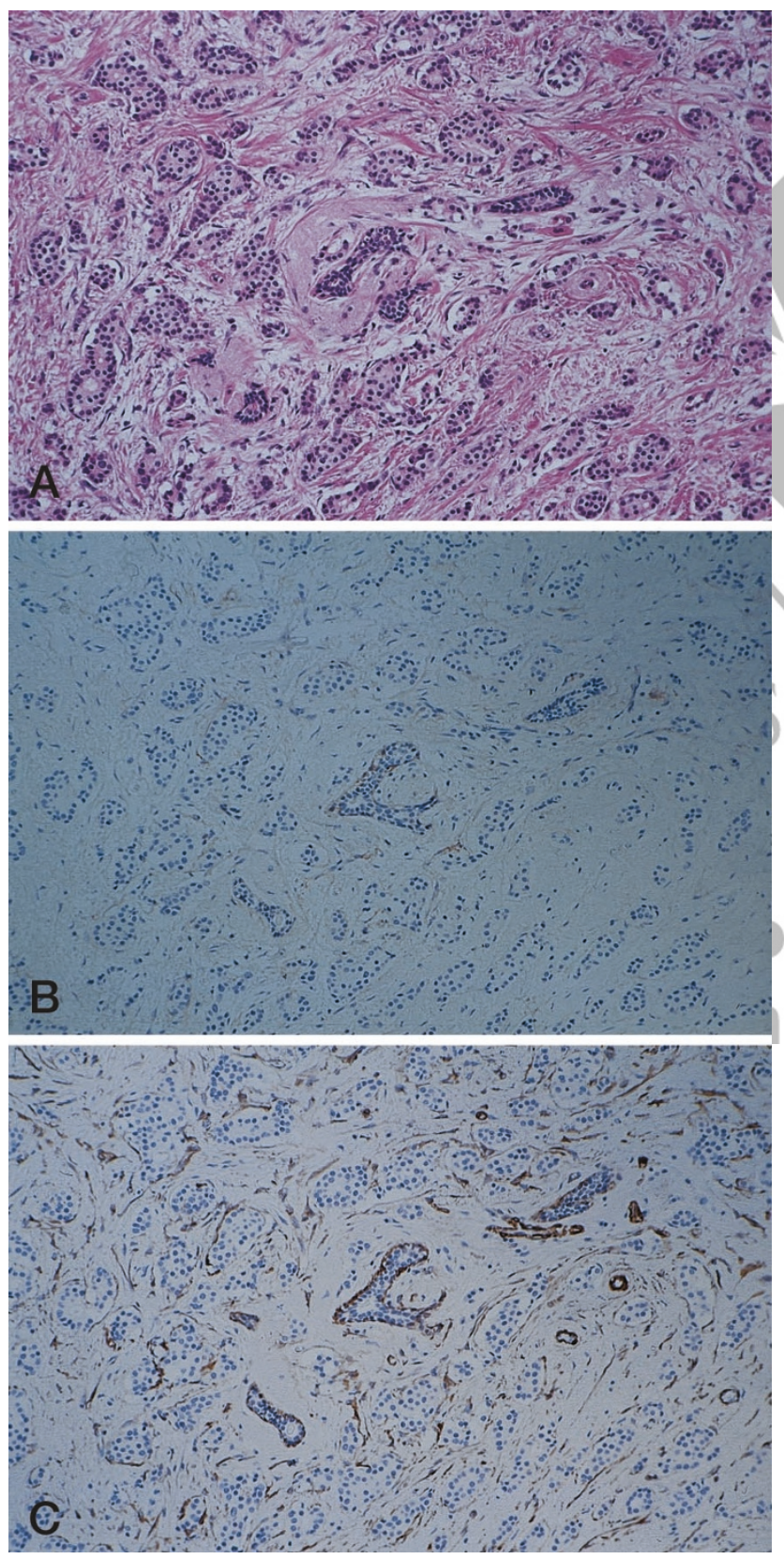

FIGURE 5. Invasive ductal carcinoma (A). CD10 (B) was positive only in myoepithelial cells of a few nonneoplastic glands entrapped in the tumor. Meanwhile, background staining of SMA (C) occasionally encircled the neoplastic cell nests.
Fibroadenoma and Phyllodes Tumor

CD10 and SMA were strongly and consistently positive in the myoepithelial cell components of both fibroadenoma and phyllodes tumor, but epithelial cells were completely negative. S-100 was positive in $50-70 \%$ of the myoepithelial cells of both types of tumors.

\section{DISCUSSION}

In the current study, we found that CD10 was consistently positive in the myoepithelial cells of the normal breast on paraffin sections. The staining pattern was identical to that of SMA. We consider that CD10 could be another useful marker of myoepithelial cells of the breast on paraffin sections. The staining pattern of S-100 was heterogenous; acinar myoepithelial cells were more frequently positive than ductal myoepithelial cells. S-100 positivity was not always specific to myoepithelial cells but was occasionally detected in luminal epithelial cells.

In ductal adenoma, adenosis, cystic glands, ductal hyperplasia, fibroadenoma, and phyllodes tumor, CD10-staining patterns of myoepithelial cells were almost identical to those of SMA. CD10 is also considered to be useful in the distinction between adenosis and invasive ductal carcinoma or between ductal adenoma and ductal carcinoma. S-100 is considered not to be very useful in practical differential diagnosis of the breast compared with CD10 and SMA; however, S-100 may play a supplementary role in some situations, such as in differential diagnosis of ductal adenoma and carcinoma.

CD10 immunohistochemistry seems to offer excellent information, especially in differential diagnosis of intraductal papillary lesions. As SMA is also positive in the vascular wall, the fibrovascular core of intraductal papillary lesions contains many SMApositive components. In complex papillary lesions that had delicate narrow fibrovascular stroma, it was difficult to find out whether the SMA-positive component adjacent to the lining epithelium was the myoepithelial cells or the thin vascular wall. Meanwhile, CD10 was completely negative in the vascular wall and clearly highlighted the myoepithelial cells. We conclude that CD10 can offer a more exact information about presence or absence of myoepithelial component than SMA when papillary lesion has a delicate complex architecture. In one case of intraductal papilloma that had both infarcted and sclerotic areas, CD10 highlighted the silhouette of the necrotic tubules. Because there was only one case studied, this may not allow a firm conclusion; however, CD10 may be helpful in spec- 
ulating whether a totally infarcted intraductal papillary lesion had been benign intraductal papilloma or intraductal papillary carcinoma when it was viable. This must be further studied by investigations involving more cases.

In atypical ductal hyperplasia and DCIS, both SMA and CD10 highlighted totally or focally retaining myoepithelial cells at the circumference of the ducts or acini that were involved by tumor. When either of them are stained positively in the circumference of acini, it seems to be useful in making a distinction between intralobular extension of DCIS and microinvasion (19). On the other hand, when both of them are negative, it is difficult to interpret it as microinvasion because not all DCIS lesions retain myoepithelial cells in their circumference as well as basement membrane (20). There was a tendency for SMA-positive area to predominate over CD10-positive area; some CD10-negative tumor cell nests showed SMA positive. It is not clear whether SMA has a higher sensitivity to myoepithelial cells or CD10 has a higher specificity to myoepithelial cells.

There are occasionally spaces between tumor cell nests and surrounding stroma. In such a situation, distinction between simple artificial spaces around the tumor and vessel involvement of the tumor becomes part of the problem. In invasive carcinomas such as invasive micropapillary carcinomas (21), SMA seems to be useful for highlighting vessel walls. However, it seems not to be useful for making the distinction between DCIS surrounded by artificial spaces and vessel involvement because SMA is positive both in myoepithelial cells and vessels. In such a situation, this distinction will be possible if CD10 is added because CD10 is totally negative in vessels and positive in myoepithelial cells.

We saw weak and scattered CD10-positive spindle stromal cells, which might be myofibroblasts, as reported elsewhere on frozen sections (9). As this CD10-positive population was only a minor component, it is not likely to cause noise on identifying myoepithelial cells. However, much more stromal cell components were positive for SMA. Especially in invasive ductal carcinomas with desmoplastic stroma, the background SMA staining of stromal cells was strong. When careful evaluation of myoepithelial cells is necessary, such as in the cases of intraductal carcinoma in sclerosing adenosis (22, 23) and lobular cancerization (24) of DCIS, CD10 seems to be better for demonstrating myoepithelial cells because the stromal background staining is weaker than that of SMA.

In summary, we concluded that CD10 could be an useful marker of myoepithelial cells on paraffin sections. Combining SMA and CD10 will allow more sophisticated evaluation of myoepithelial cells for differential diagnosis of breast lesions.

\section{REFERENCES}

1. McCarty KS, Tucker JA. Breast. In: Sternberg SS, editor. Histology for pathologists. New York: Raven; 1992. p. 893-902.

2. Rosen PP, editor. Breast pathology, diagnosis by needle core biopsy. Philadelphia: Lippincott; 1999.

3. Carter D, Orr SL, Merino MJ. Intracystic papillary carcinoma of the breast. After mastectomy, radiotherapy or excisional biopsy alone. Cancer 1983;52:14-9.

4. Lefkowitz ML, Usar CM, Lefkowitz W, Wargotz ES. Intraductal (intracystic) papillary carcinoma of the breast and its variants: a clinicopathological study of 77 cases. Hum Pathol 1994;25:802-9.

5. Rosen PP. Microglandular adenosis. A benign lesion simulating invasive mammary carcinoma. Am J Surg Pathol 1983; 7:137-44

6. Tavassoli FA, Norris HJ. Microglandular adenosis of the breast. A clinicopathologic study of 11 cases with ultrastructural observations. Am J Surg Pathol 1983;7:731-7.

7. Tsuda H, Takarabe T, Hasegawa T, Murata T, Hirohashi S. Myoepithelial differentiation in high-grade invasive ductal carcinomas with large central acellular zones. Hum Pathol 1999;30:1134-9.

8. Metzgar RS, Borowitz MJ, Jones NH, Dowell BL. Distribution of common acute lymphoblastic leukemia antigen in nonhematopoietic tissues. J Exp Med 1981;154:1249-54.

9. Mechtersheimer G, Möller P. Expression of the common acute lymphoblastic leukemia antigen (CD10) in mesenchymal tumors. Am J Pathol 1989;134:961-5.

10. Sato $\mathrm{Y}$, Itoh F, Hinoda $\mathrm{Y}$, Ohe $\mathrm{Y}$, Nakagawa N, Ueda R, et al. Expression of CD10/neutral endopeptidase in normal and malignant tissue of the human stomach and colon. J Gastroenterol 1996;31:12-7.

11. Chu P, Arber DA. Paraffin section detection of CD10 in 505 nonhematopoietic neoplasms. Frequent expression in renal cell carcinoma and endometrial stromal sarcoma. Am J Clin Pathol 2000;113:374-82.

12. Avery AK, Beckstead J, Renshaw AA, Corless CL. Use of antibodies to RCC and CD10 in the differential diagnosis of renal neoplasms. Am J Surg Pathol 2000;24:203-10.

13. Yaziji H, Gown AM. Immunohistochemical analysis of gynecologic tumors. Int J Gynecol Pathol 2001;20:64-78.

14. Chu PG, Arber DA, Weiss LM, Chang KL. Utility of CD10 in distinguishing between endometrial stromal sarcoma and uterine smooth muscle tumors: an immunohistochemical comparison of 34 cases. Mod Pathol 2001;14:465-71.

15. Letarte M, Vera S, Tran R, Addis JBL, Onizuka RJ, Quackenbush EJ, et al. Common acute lymphocytic leukemia antigen is identical to neutral endopeptidase. J Exp Med 1988;168: 1247-53.

16. Gusterson BA, Monaghan P, Mahendran R, Ellis J, O'Hare MJ. Identification of myoepithelial cells in human and rat breasts by anti-common acute lymphoblastic leukemia antigen antibody A12. J Natl Cancer Inst 1986;77:343-9.

17. Clarke C, Titley J, Davies S, O'Hare MJ. An immunomagnetic separation method using superparamagnetic (MACS) beads for large-scale purification of human mammary luminal and myoepithelial cells. Epithelial Cell Biol 1994;3:38-46.

18. Mechtersheimer G, Krüger KH, Born IA, Möller P. Antigenic profile of mammary fibroadenoma and cystosarcoma phyllodes. A study using antibodies to estrogen- and progesterone receptors and to a panel of cell surface molecules. Pathol Res Pract 1990;186:427-38.

19. Prasad ML, Hyjek E, Giri DD, Ying L, O'Leary JJ, Hoda SA. Double immunolabeling with cytokeratin and smooth- 
muscle actin in confirming early invasive carcinoma of breast. Am J Surg Pathol 1999;23:176-81.

20. Rajan PB, Perry RH. A quantitative study of patterns of basement membrane in ductal carcinoma in situ. Breast J 1995;1:315-21.

21. Siriaunkgul S, Tavassoli FA. Invasive micropapillary carcinoma of the breast. Mod Pathol 1993;6:660-2.
22. Chan JKC, Ng WF. Sclerosing adenosis cancerized by intraductal carcinoma. Pathology 1987;19:425-8.

23. Oberman HA, Markey BA. Non-invasive carcinoma of the breast presenting in adenosis. Mod Pathol 1991;4:31-5.

24. Kerner H, Lichtig C. Lobular cancerization: incidence and differential diagnosis with lobular carcinoma in situ of breast. Histopathology 1986;10:621-9.

\section{Book Review}

Dabbs DJ, editor: Diagnostic Immunohistochemistry, 685 pp, London, Churchill Livingstone, 2002 (\$269.00).

This rather voluminous textbook (more than 650 pages) summarizes in an encyclopedic fashion almost everything that is currently known about the immunoreactivity patterns of human tumors for the several hundreds of antibodies in use in immunohistology laboratories. The book offers a wealth of information in the form of written text, many micrographs that are often (but not always) well chosen and of good quality, bar graphs and tables, diagnostic algorithms or rather decision trees, and thoūsands of references.

The opening chapter, on techniques, is excellent. It is concise yet comprehensive, systematic yet practical in everyday use. The second chapter, on cost effectiveness, which constitutes with the first the methodological introduction to the organ system-oriented chapters, disserves the book. I simply could not follow the authors' lines of reasoning. What, for example, to make of the following phrase 'One reason why immunohistochemistry appears to be so cost effective is that the benefit to cost ratio of immunohistochemistry is relatively low compared with the same ratio in other medical procedures.'

Things get better in the organ-oriented chapters but to a variable degree. An overall characteristic of the book is the variability in approach and in didactic quality between the chapters. This is frequently the case in multi-authored volumes and might have been avoided by much more rigorous editing. Some chapters are of exemplary quality. The chapter on non-Hodgkin's lymphomas is concise and practice-oriented. The key diagnostic points are a real help in diagnoses. The chapter on soft tissue and osseous neoplasms, in contrast, is very descriptive, has no key diagnostic points, and might not be a big help in solving diagnostic problems. Some chapters lack accuracy in the description of the characteristics of the relevant antigens. About laminin I read, 'It is a $1000 \mathrm{kd}$ molecule, the three short forms and one long arm of which have globular end regions.' That it is a family of heterotrimeric cross-shaped molecules, of which some members show cell type specific localization (which might even be of diagnostic use!), is left to the imagination of the author.

The diagnostic algorithms are a nice idea but difficult/in use. Where do they begin? How reliable is their use (as an immunoreactivity-guided decision tree) in diagnostic practice?

A striking example of the heterogeneity of the book is the printing of the graphs and the algorithms. Some chapters have the graphs in color, others in black and white, and this holds true also for the algorithms.

= Overall, therefore, I have somewhat mixed feelings about the book. The wealth of information is its major asset: if you know what you are looking for and are persistent, you will probably find it. If you have a problem in your daily diagnostic practice, the book might appear less helpful than you hoped. Time will tell whether in its present form it will become a classic. I wonder.

Fred Bosman

University of Lausanne

Lausanne, Switzerland 